\title{
Assessing outcome of out-of-hospital cardiac arrest due to subarachnoid hemorrhage using brain CT during or immediately after resuscitation
}

YOSHIHIRO MORIWAKI $(\bowtie) \cdot$ NORIYUKI SUZUKI• HIROSHI MANAKA • KOICHI HAMADA

Critical Care and Emergency Center Yokohama City University Medical Center 4-57 Urafune-cho, Minami-ku Yokohama, Japan 232-0024

Phone: +81-45-261-5656 Fax: +81-45-2433677 E-mail: qqc3@yokohama-cu.ac.jp

\section{NORIYUKI SUZUKI • YOSHIHIRO MORIWAKI • HIROSHI MANAKA • KOICHI HAMADA}

\begin{abstract}
Objectives. The clinical course and outcome of out-of-hospital cardiopulmonary arrest (OHCPA) due to subarachnoid hemorrhage (SAH) is unclear. The objective of this study is to clarify them.

Study design. Single- center, observational study.

Setting. We usually perform a brain computed tomography (CT) in OHCPA patients who present without a clear etiology (42\% of all OHCPA), such as trauma, to determine the cause of OHCPA and to guide treatment.

Patients. The study included OHCPA patients without a clear etiology, who were transferred to our center and who underwent a brain $\mathrm{CT}$ during resuscitation.

Methods of measurement. Patients' records were reviewed; initial cardiac rhythm, existence of a witness and bystander cardiopulmonary resuscitation efforts (CPR) were compared with patients' outcomes.

Results. Sixty-six patients were enrolled. $72.7 \%$ achieved return of spontaneous circulation (ROSC), $71.2 \%$ were admitted, $30.3 \%$ survived more than 7 days, and 9.1. survived-to-discharge. In 41 witnessed OHCPA, 87.8\% obtained ROSC, 85.4\% were admitted, and $14.6 \%$ survived-to-discharge. All survivors were witnessed. In 25 non-witnessed OHCPA, $48 \%$ obtained ROSC and were admitted, and no patients were discharged. Initial cardiac rhythm was ventricular fibrillation (VF), pulseless electrical activity (PEA) and asystole in 3.0\%,39.4\%, and 47.0\%. In 2 VF patients $50.0 \%$ survived-to- discharge, and there was no survivor with PEA or asystole.

Conclusion. This study shows a high rate of ROSC and admission in OHCPA patients with a SAH, and also reveals their very poor neurological outcome. We conclude that the detection of a SAH in OHCPA patients is important to determine the accurate frequency of SAH in this patient group and to guide appropriate treatment of all OHCPA patients.
\end{abstract}

Keywords: out-of-hospital cardiac arrest, brain CT, outcome, autopsy imaging, postmortem imaging

\section{Introduction}

Usually, outcomes for out-of-hospital cardiopulmonary arrest (OHCPA) patients are poor. Although a witnessed OHCPA of cardiac origin, with ventricu- lar fibrillation (VF) on the scene at the arrival of the ambulance, is considered to carry a comparably favorable prognosis, patients with OHCPA due to noncardiac origin typically have a dismal chance of survival. On the contrary, we often encounter OHCPA patients due to non-cardiac origin, such as a subarachnoid hemorrhage $(\mathrm{SAH})$, presenting with
VF and some signs of cardiac diseases on electrocardiogram or echocardiography during their resuscitation process. Or, we often encounter OHCPA patients who successfully achieve return of spontaneous circulation (ROSC), and whose brain computed tomography (CT) after ROSC disclose that the cause of CPA was a SAH. However, it is still 
Table 1. The outcome of subgroups of patients: the rate of return of spontaneous circulation (ROSC), who was admitted, and who survived to discharge: witnessed vs. non-witnessed, and bystander cardiopulmonary resuscitation (BS-CPR) vs. no BS-CPR, there was no statistical difference between witnessed and non-witnessed, and between BS-CPR and no BS-CPR. Data means sum of patients more favorable than their category, and () means percentage per all cases in each subgroup.

\begin{tabular}{lllll}
\hline & Witnessed & Non-witnessed & Total & \\
\hline All cases & 41 & 25 & 66 & \\
ROSC & $36(87.8 \%)$ & $12(48.0 \%)$ & $48(72.7 \%)$ & \\
$\begin{array}{l}\text { Admitted } \\
\text { survive- }\end{array}$ & $35(85.4 \%)$ & $12(48.0 \%)$ & $47(71.2 \%)$ & \\
to-discharge & $6(14.6 \%)$ & 0 & $6(9.1 \%)$ & \\
\hline & BS-CPR & no BS-CPR & BS-CPR & no BS-CPR \\
\hline All cases & 27 & 14 & 7 & 18 \\
ROSC & $24(88.9 \%)$ & $12(85.7 \%)$ & $6(85.7 \%)$ & $6(33.3 \%)$ \\
Admitted & $23(85.2 \%)$ & $12(95.7 \%)$ & $6(85.7 \%)$ & $6(33.3 \%)$ \\
survive- & $5(18.5 \%)$ & $1(7.1 \%)$ & 0 & 0 \\
to-discharge & & 0.934 & 0.891 & \\
\hline p value & & & & 0 \\
\hline
\end{tabular}

BS-CPR, bystander cardiopulmonary resuscitation; ROSC, return of spontaneous circulation.

Table 2. The rate of return of spontaneous circulation (ROSC), who were admitted, and who survived to discharge, there was no statistical difference among initial cardiac rhythm. Data means sum of patients more favorable than their category, and () means percentage per all cases in each subgroup.

\begin{tabular}{llll}
\hline & VF & Asystole & PEA \\
\hline All cases & 2 & 31 & 26 \\
ROSC & $1(59 \%)$ & $17(54.8 \%)$ & $24(92.3 \%)$ \\
Admitted & $1(59 \%)$ & $17(54.8 \%)$ & $23(76.9 \%)$ \\
survived-to-discharge & $1(59 \%)$ & $2(6.5 \%)$ & $0(0 \%)$ \\
\hline p value & & 0.261 & \\
\hline
\end{tabular}

PEA, pulseless electrical activity, ROSC, return of spontaneous circulation; VF, ventricular fibrillation.

unclear what the outcome of OHCPA due to non-cardiac origin, particularly SAH will be. There are only a few reports concerning the clinical course and outcome in these patients, (1) so we cannot accurately evaluate the incidence of SAH (using brain CT during and immediately after resuscitation).

One of the reasons why we had limited these examinations cannot change the patient's prognosis and instead, chest compressions are a top priority throughout the resuscitation process. In many hospitals in J apan, including our hospital, because of the short distance between the emergency department and the CT room, $(2,3)$ some imaging, such as ultrasonography (US) and $\mathrm{CT}$, is performed during resuscitation. We can detect a SAH using CT during resuscitation in OHCPA patients, observe these patients and their outcome. In this study, we tried to clarify the clinical course and outcome of OHCA due to SAH.

\section{Materials and methods \\ Study design \\ This study is a single center, observa- tional study.}

\section{Setting}

In our emergency department (ED), we usually perform echocardiography and abdominal sonography, chest x-ray, blood examination, including Troponin I or $\mathrm{T}$, and brain or chest $\mathrm{CT}$ to determine the cause of OHCPA and to treat this pathological condition in patients without clear etiology such as trauma, hanging, drowning, asphyxia and terminal stage of some disease. We performed brain CT in $42 \%$ of all OHCPA patients. (1) We have established a pre-hospital and inter-hospital Emergency Medical Service (EMS) system for severe patients including OHCPA in our city, Yokohama (the surface area is $434 \mathrm{Km}^{2}$ and the population is 3.37 million). We selected 11 hospitals, which can receive and treat the most severe patients, including OHCPA patients. The emergency director (a medical doctor) conducts his duties in the Central Operations Center, in the fire department of the city, where he notifies the hospitals of the transfer of OHCPA patients. In Yokohama, data on OHCPA patients is thought to be population based. $(2,4,5)$

Selection of participants

The study included non-traumatic OHCPA patients without a clear etiolo- 
gy such as trauma, hanging, drowning, asphyxia and terminal stage of some disease, who were transferred to our center and underwent brain CT during resuscitation.

\section{Methods of measurement}

Emergency department patient records were reviewed; initial cardiac rhythm, presence of VF during treatment, existence of a witness and bystander cardiopulmonary resuscitation efforts (CPR) were compared with patient outcomes.

\section{Results}

Sixty-six patients were enrolled. Forty one arrests were witnessed and 34 underwent bystander CPR. Forty eight (72.7\%) achieved ROSC, 47 (71.2\%) were admitted, 20 (30.3\%) survived more than 7 days, and $6(9.1 \%)$ survived-to-discharge, of whom 3 were in a severe condition and 3 were in a vegetative state. There was no neurologically intact survivor. In 41 witnessed OHCPA, $36(87.8 \%)$ obtained ROSC, 35 (85.4\%) were admitted, and $6(14.6 \%)$ survived-to-discharge. All patients who survived had witnesses. On the contrary, in 25 non-witnessed OHCPA 12 (48.0\%) obtained ROSC and were admitted but no patients were discharged (table 1). There was no statistical difference in the rate of ROSC, admission and survival-to-discharge between witnessed and non-witnessed patients. Initial cardiac rhythm was VF, pulseless electrical activity (PEA) and asystole in $2(3.0 \%), 26(39.4 \%)$, and 31 (47.0\%) patients, respectively. Nine patients $(13.6 \%)$ showed VF during resuscitation. In 2 VF patients 1 (50.0\%) survived-to-discharge, and there were no survivors among 26 PEA patients and 31 asystole patients (table 2 ). There was no statistical difference in the rate of ROSC, admission and survival-todischarge among these rhythms.

\section{Discussion}

It is well known that SAH is one of the common causes of OHCPA. $(6,7)$ The true incidence of SAH as a cause of CPA is difficult to determine. One clini- cal study showed that only $3.5 \%$ of OHCPA survivors had an aneurismal SAH (6) and another showed SAH was observed in $16.2 \%$ of hemodynamically stable non-traumatic OHCPA survivors who und erwent a brain $\mathrm{CT}$, whose Fisher SAH grade was either III or IV. (8) One of the reasons why it is difficult to determine the rate of SAH in OHCPAs is that brain CTs of OHCPA survivors or non-survivors is rarely performed immediately after resuscitation or during resuscitation. Classically, we can determine the cause of OHCPA only by autopsy, if patients cannot be resuscitated or die shortly after ROSC, but the frequency of autopsy overall is usually very low and unsatisfactory, even today in developed countries. Recently, postmortem imaging or autopsy imaging has been discussed as a possible substitute for autopsies to determine the cause of CPA.

Although CT scanners are wide spread in many hospitals nowadays, CT is not routinely used in unconscious patients. Particularly for OHCPA patients and post-resuscitated patients, CT is not routinely performed even in high quality emergency departments in developed countries. We have limited data on OHCPA patients, due to SAH, only for those who are successfully resuscitated and then undergo a brain CT. This data can never indicate accurately the clinical features of the whole course OHCPA due to SAH. In our hospital, we performed $\mathrm{CT}$ for OHCPA patients during resuscitation without clear etiology. We reported in our previous study that brain CTs were performed on 293 patients out of 698 non-traumatic and non-neck-hanging OHCPA patients. However, these 698 patients included OHCPA patients with a clear etiology who were not intended for a brain CT. In this study we showed more reliable data than in the previous study which dealt with very restricted materials. (1) The clinical features, course and outcome of OHCPA due to SAH described in this report is thought to be reliable. Althoughsome previous studies showed that the survival rate of OHCPA patients due to SAH was lower compared with that due to non-SAH, another author reported a high survival rate of $19 \%$ of respiratory arrest survivors due to $\mathrm{SAH}$. (9) Our study showed that OHCPA patients, due to $\mathrm{SAH}$, had a high survival rate and adequate chance to undergo surgical procedures, such as clipping and transarterial coiling. It is important to detect SAH in OHCPA patients using brain $\mathrm{CT}$ immediately after resuscitation both in order to prevent the worsening effect of some treatments for acute coronary syndromes and in order to give the patient a chance to undergo appropriate treatments for $\mathrm{SAH}$ due to a ruptured aneurism. It is also important to detect SAH in OHCPA patients, even if they cannot obtain ROSC, using brain $\mathrm{CT}$ during resuscitation in order to evaluate the exactepidemiology of SAH from the view point of public health in each local area.

OHCPA due to SAH often presents abnormal electrocardiogram (ECG) findings such as ST elevation, negative $T$ and other ST-T changes, which are usually seen in patients with acute coronary syndromes or pulmonary embolism. It has been known that SAH can be responsible for many cardiopulmonary pathophysiological conditions. $(10,11)$ The pathophysiology of SAH patients with CPA has been attributed to a sudden increase in intracranial pressure with brainstem herniation and respiratory arrest or to an excessive release of catecholamine resulting in VF and other cardiac abnormalities. (11) Ourstudy, however, indicated lower frequency of VF and other abnormal ECG findings in the resuscitation process of OHCPA due to SAH than reported in previous studies. In OHCPA patients, there may be little response to $\mathrm{SAH}$ including release of catecholamines. Unfortunately, most treatments for acute coronary syndromes or pulmonary embolism might worsen SAH. Noritomi reported that some doctors are not very aware of the possibility of the diagnosis of SAH within the context of CPA.(12) However, owing to routine brain scanning, we did not treat our patients with OHCPA due to SAH as acute coronary syndromes, even if 
they present VF during resuscitation process. Many authors have emphasized that diagnosis of SAH in OHCPA patients might increase the safety of management for these patients, who could undergo aggressive interventions such as embolization or surgical clipping after successful resuscitation to prevent aneurismal rerupture. $(13,14)$ We conclude that this study shows a high rate of ROSC and admission in OHCPA patients due to $\mathrm{SAH}$, and also shows that their neurological outcome is very poor. We conclude that detection of SAH in OHCPA patients is important to accurately determine the frequency of SAH in OHCPAs and to improve the treatment of these patients.

\section{REFERENCES}

1. Moriwaki Y, Tahara Y, Arata S, Toyoda H, Iwashita M, Kosuge T, et al. Etiology of out-of-hospital cardiac arrest due to non-cardiac origin in J apan. Intensive Care Med 2009;35:S144.

2. Moriwaki Y, Sugiyama M, Hayashi H, Mosiello G, Cremonese F, Altomani V, et al. Emergency medical service system in Yokohama, J apan. Annali Degli Ospedali San Camillo e Forlanini 3 2001;34456.

3. Moriwaki Y, Sugiyama M, Toyoda H, Kosuge T, Takahashi K, Iwashita M, et al. Monitoring and evaluation of intraperitoneal bleeding (IPB) by small portable ultrasonography during transcatheter arterial embolization (TAE) in abdominal-pelvic trauma patients with shock: as a monitor for early detection of increase of IPB. Hepatogastroenterol 2006;53:175-8.

4. Moriwaki Y, Sugiyama M, Toyoda H, Kosuge T, Iwashita M, Ishikawa J, et al. Bystander CPR for out-of-hospital cardiac arrest in J apan. Crit Care 2007;11:S130-1

5. Moriwaki Y, Sugiyama M, Suzuki N. Outcome of blunt traumatic cardiopulmonary arrest. Intensive Care Med 2007;33:S269.

6. Kurkciyan I, Meron G, Sterz F, Domanovits H, Tobler K, Laggner AN, et al. Spontaneous subarachnoid hemorrhage as a cause of out-ofhospital cardiac arrest. Resuscitation 2001;51:27-32.

7. Schievink WI, Wijdicks EF, Parisi J E, Piepgras DG, Whisnant JP. Sudden death from aneurismal subarachnoid hemorrhage. Neurology 1995;45:871-4

8. Inamasu J, Miyatake S, Tomioka H, Suzuki M, Nakatsukasa M, Maeda N, et al. Subarachnoid hemorrhage as a cause of out-of-hospital cardiac arrest: a prospective computed tomography study. Resuscitation 2009;80:977-80.

9. Shapiro S. Management of subarachnoid hemorrhage patients who presented with respiratory arrest resuscitated with bystander CPR. Stroke 1996;27:1780-2.

10. Macrea LM, Tramer MR, Walder B. Spontaneous subarachnoid hemorrhage and serious cardiopulmonary dysfunction? A systematic review. Resuscitation 2005;65:139-48.

11. Tabbaa MA, Ramirez-Lassepas M, Snyder BD. Aneurismal subarachnoid hemorrhage presenting as cardiorespiratory arrest. Arch Intern Med 1987;147:1661-2.

12. Noritomi DT, de Cleva R, Beer I, Dalbem AG, Liborio AB, Frota NA, et al. Doctor's awareness of spontaneous subarachnoid hemorrhage as a cause of cardiopulmonary arrest. Resuscitation 2006;71:123-4.

13. Inamasu J , Saito R, Nakamura Y, Ichikizaki K, Suga S, Kawase T, et al. Survival of a subarachnoid hemorrhage patient who presented with prehospital cardiopulmonary arrest: case report and review of the literature. Resuscitation. 2001;51:207-11.

14. Hess EP, Boie ET, White RD. Survival of a neurologically intact patient with subarachnoid hemorrhage and cardiopulmonary arrest. Mayo Clin Proc. 2005;80:1073-6. 